\title{
Local thermodynamic equilibrium in a laser-induced plasma evidenced by blackbody radiation
}

\author{
Jörg Hermann $^{\mathrm{a}, *}$, David Grojo ${ }^{\mathrm{a}}$, Emanuel Axente ${ }^{\mathrm{b}}$, Valentin Craciun ${ }^{\mathrm{b}}$ \\ ${ }^{a}$ Aix-Marseille University, CNRS, LP3, 13009 Marseille, France \\ ${ }^{b}$ National Institute for Lasers, Plasma and Radiation Physics, 77125 Măgurele, Romania
}

\begin{abstract}
We show that the plasma produced by laser ablation of solid materials in specific conditions has an emission spectrum that is characterized by the saturation of the most intense spectral lines at the blackbody radiance. The blackbody temperature equals the excitation temperature of atoms and ions, proving directly and unambiguously a plasma in local thermodynamic equilibrium. The present investigations take benefit from the very rich and intense emission spectrum generated by ablation of a nickel-chromium-molybdenum alloy. This alternative and direct proof of the plasma equilibrium state re-opens the perspectives of quantitative material analyses via calibration-free laser-induced breakdown spectroscopy. Moreover, the unique properties of this laser-produced plasma promote its use as radiation standard for intensity calibration of spectroscopic instruments.
\end{abstract}

Keywords: Blackbody radiation; Local thermodynamic equilibrium; LIBS; Calibration-free.

\section{Introduction}

The size of laboratory plasmas being usually smaller than their characteristic length of radiation relaxation, the state of complete thermodynamic equilibrium is generally not established. Most of the generated photons escape from the plasma and only a minor fraction of them is reabsorbed. In atmospheric plasmas, the collision rates are large enough so that the characteristic lengths of particle relaxation are typically smaller than the plasma size [1]. This favors the establishment of local thermodynamic equilibrium: the plasma state can then be modeled in a simplified way using the statistical laws of equilibrium $[2,3]$. However, as the characteristic times of particle relaxation and particle diffusion are similar, atmospheric density plasmas have nonuniform spatial distributions of temperature and densities [4, 5]. Plasmas produced by laser ablation of solids differ from other atmospheric plasmas [6]. According to their large initial density, the processes of relaxation are faster whereas the diffusion processes are slower $[7,8]$. Due to the high initial pressure, the ablated material plume expands rapidly until it reaches a pressure equilibrium with the surrounding gas atmosphere $[9,10]$. It was

${ }^{*}$ Corresponding author: hermann@lp3.univ-mrs.fr shown that for laser irradiation with ultraviolet nanosecond laser pulses, the plasma appears almost uniform if ablation occurs under an inert gas atmosphere [11]. The combination of both local thermodynamic equilibrium (LTE) and uniform spatial distribution qualifies such a plasma as an ideal radiation source that was evidenced by several signatures in the emission spectra of plumes produced by laser ablation of different materials [12]. In the present research note, we focus on a particular feature of such an ideal radiation source. Although the characteristic length of radiation relaxation is expected to exceed the plasma dimension for most wavelengths, the situation can be different for photons emitted by strong resonance lines. Their probability of being absorbed within the plasma is expressed by the optical thickness $\tau=\int \alpha(z) d z$, where $\alpha$ is the absorption coefficient and $z$ the coordinate along the line of sight. In the case of LTE, the processes of absorption and emission are related through Kirchhoff's law of thermal radiation $\varepsilon_{\lambda} / \alpha=U_{\lambda}$. Here, $\varepsilon_{\lambda}$ is the spectral emission coefficient and $U_{\lambda}$ is the blackbody spectral radiance given by Planck's law. For a uniform spatial distribution, the spectral radiance of the plasma in LTE is given by [13]

$$
B_{\lambda}=U_{\lambda}\left(1-e^{-\tau}\right) .
$$

Here $\tau=\alpha L$, where $L$ is the plasma size along the line of sight. 
In case of radiation for which the characteristic length of absorption is much smaller than the plasma size $(\tau>>$ 1), Eq. (1) simplifies to

$$
B_{\lambda}^{\text {thick }}=U_{\lambda} \text {. }
$$

When Eq. (2) is fulfilled for the entire spectrum the plasma is in complete thermodynamic equilibrium.

The most famous blackbody radiator is the Sun. According to its surface temperature of about $5800 \mathrm{~K}$, the emission spectrum has an intensity maximum located in the yellow-green spectral range at $500 \mathrm{~nm}$ [14]. In metallurgy, the blackbody radiation from metal surfaces is explored to measure the temperature. These pyrometric measurements take advantage of the temperaturedependence of the blackbody emission maximum predicted by Wien's law [15].

Blackbody radiation has been rarely observed from laboratory plasmas. According to their small size, the conditions of strong optical thickness are hardly achieved. The observation also implies experimental difficulties as it requires the acquisition of a large spectral range with an intensity-calibrated spectroscopic apparatus.

During the early development of equilibrium plasma sources, the blackbody radiation limit was often mentioned but rarely demonstrated by measurements [4]. As an example, the blackbody radiation limit was evidenced in the vacuum ultraviolet range for an argon arc [16]. The saturation of a helium resonance line at the blackbody radiance was observed in a shock-tube experiment [17]. The plateau at the blackbody radiance of strong $\mathrm{Ti}^{+}$-transitions was also noticed for short times $(t<200 \mathrm{~ns})$ during laser ablation of titanium in a lowpressure nitrogen atmosphere [18].

In the present experiment, we explore the very rich emission spectrum of a vaporized nickel-chromiummolybdenum alloy to visualize the blackbody radiance for the plasma produced by laser ablation with UV nanosecond pulses in argon background gas. These conditions have been previously identified to generate an ideal radiation source [12] and the present measurements consolidate the former results illustrating the remarkable properties of this source.

\section{Experiment}

The experiments were carried out in a temperaturestabilized laboratory environment using a frequencyquadrupled Nd:YAG laser source (Quantel, model Brilliant). The laser delivers ultraviolet $(266 \mathrm{~nm}$ ) pulses of $4 \mathrm{~ns}$ duration. The pulse energy was attenuated down to $6 \mathrm{~mJ}$. After beam focusing with a lens of
Table 1: Composition of the nickel-chromium-molybdenum alloy (Hart b.v., Inconel 625) : mass fractions given by the manufactor $C_{\text {manufac }}$ and values deduced from the LIBS spectra analysis $C_{L I B S}$.

\begin{tabular}{ccc}
\hline Element & $C_{\text {manufac }}(\%)$ & $C_{\text {LIBS }}(\%)$ \\
\hline $\mathrm{Ni}$ & $58 \min$ & 63.1 \\
$\mathrm{Cr}$ & $20.0-23.0$ & 21.0 \\
$\mathrm{Mo}$ & $8.0-10.0$ & 9.4 \\
$\mathrm{Nb}$ & $3.15-4.15$ & 3.7 \\
$\mathrm{Fe}$ & 5.0 & 1.7 \\
$\mathrm{Mn}$ & $0.5 \max$ & 0.07 \\
$\mathrm{Si}$ & $0.5 \max$ & 0.35 \\
$\mathrm{Al}$ & $0.4 \max$ & 0.30 \\
$\mathrm{Ti}$ & $0.4 \max$ & 0.24 \\
$\mathrm{Co}$ & $1.0 \max$ & 0.1 \\
\hline
\end{tabular}

$150 \mathrm{~mm}$ focal length to a spot diameter of $100 \mu \mathrm{m}$, a laser fluence of about $80 \mathrm{~J} \mathrm{~cm}^{-2}$ was incident on the sample surface. The nickel-chromium-molybdenum alloy of composition given in Table 1 was placed on a motorized sample holder in a vacuum chamber of $10^{-4} \mathrm{~Pa}$ residual pressure. During the experiments, the chamber was filled with argon at $5 \times 10^{4}$ Pa pressure.

Optical emission spectroscopic measurements were performed by imaging the plasma with two lenses of 150 and $35 \mathrm{~mm}$ focal lengths onto the entrance of an optical fiber of $600 \mu \mathrm{m}$ core diameter. According to the image magnification 1:5, the entire plasma volume was observed. The fiber was coupled to an echelle spectrometer of $0.4 \mathrm{~m}$ focal length with two optical paths for the UV and visible/near infrared spectral ranges (LTB, model Aryelle Butterfly). Using an intensified charge-coupled device (ICCD) matrix detector for photon detection, a resolving power of $1.4 \times 10^{4}$ and $9.4 \times 10^{3}$ is obtained for the UV and visible spectral ranges, respectively.

The spectral resolution of the apparatus was measured as a function of wavelength using a low-pressure argon-mercury lamp. An intensity calibration of the spectroscopic apparatus was performed in the visible and UV spectral ranges using calibrated tungsten and deuterium lamps, respectively.

The spectra were recorded for different delays of the detector gate $t_{g}$ with respect to the laser pulse, setting the gate width $\Delta t_{g}$ small enough so that the variations of temperature and electron density during the time of observation were small compared to their absolute values. Typically we applied $\Delta t_{g} \leq t_{g} / 2$. To enhance the signal-to-noise ratio, data acquisition was performed by averaging over 200 ablation events, applying 10 pulses on 20 different irradiation sites. The sites were separated by a distance of $150 \mu \mathrm{m}$. 


\section{Results and discussion}

\subsection{Plasma diagnostics}

The spectra recorded for different observation times were compared to the spectral radiance of a uniform LTE plasma calculated using Eq. (1). An iterative measurement loop was applied to deduce electron density, temperature and relative elemental fractions. For details, we refer to previous work [19, 20].

The main problem of the spectra calculation relies in the low accuracy of available spectroscopic data. For example, the transition probabilities $A_{u l}$ of the most intense $\mathrm{Ni}$ and $\mathrm{Ni}^{+}$lines are given with an accuracy ranging from $25 \%$ to $50 \%$ [21]. For niobium, no $A_{u l}$-values are tabulated in the NIST database. In that case, data are taken from the Kurucz database in which the transition probabilities are reported without mentioning the accuracy [22].

To minimize the error of temperature measurements due to the low accuracy of spectroscopic data, we constructed Boltzmann plots of the major elements with a large number of spectral lines. The lines were selected according to $A_{u l}$-accuracy, measured signal-to-noise

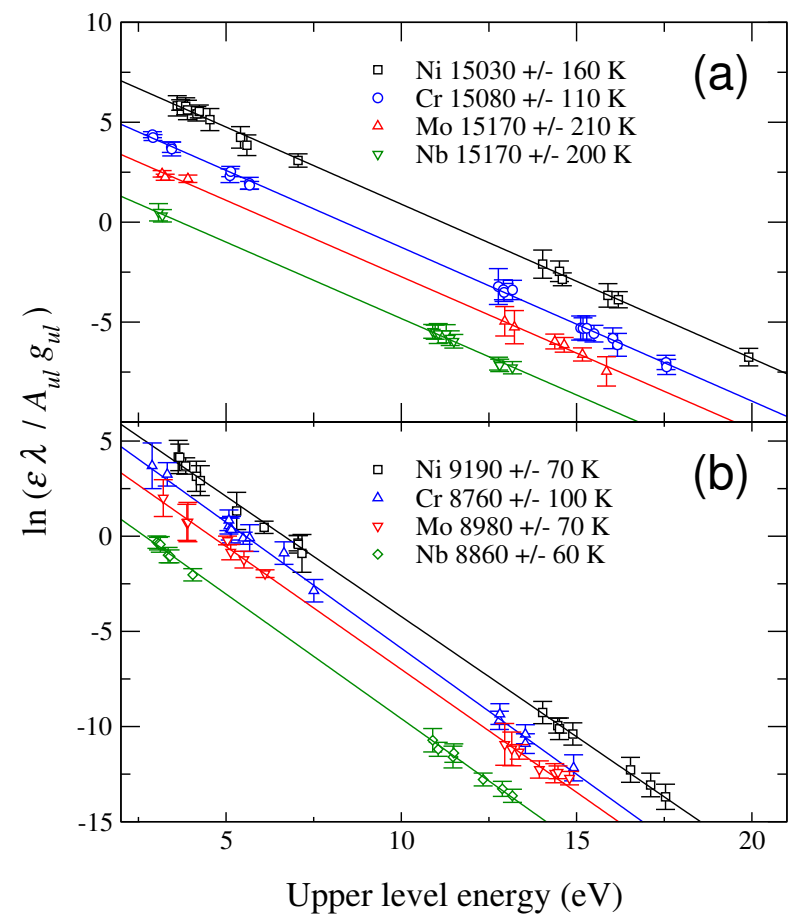

Figure 1: Saha-Boltzmann plots of major elements obtained from spectra recorded for $t=230 \mathrm{~ns}$ (a) and $2500 \mathrm{~ns}$ (b). In the logarithmic function, $\varepsilon, \lambda, A_{u l}$ and $g_{u l}$ are the emission coefficient, the wavelength, the transition probability and the upper level statistical weight of the transitions, respectively.

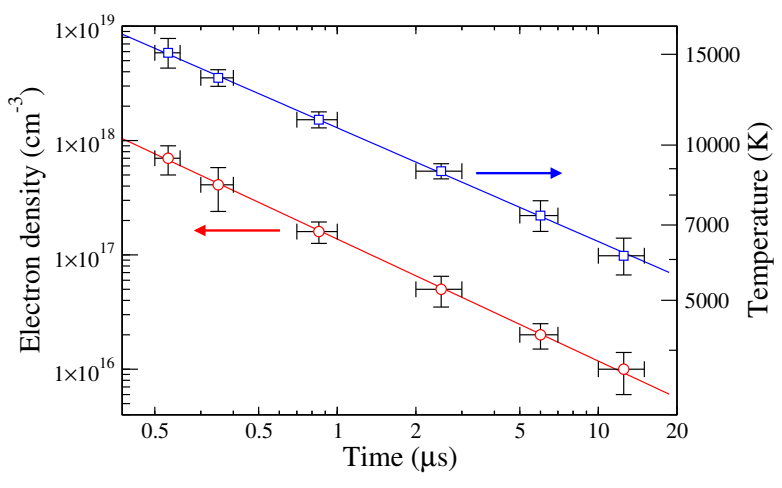

Figure 2: Electron density (circles) and temperature (squares) vs time. The vertical error bars represent the measurement uncertainties whereas the horizontal error bars indicate the time of observation (gate width).

ratio, interference with other transitions and optical thickness. As these line attributes change with the evolution of the plasma properties, the ensemble of chosen lines varies with the measurement time.

Saha-Boltzmann plots of the major sample elements (see Table 1) are displayed in Fig. 1 for two different recording times. The error bars take into account measurement uncertainties due to noise, intensity calibration and line interferences. Moreover, the accuracy of the transition probabilities and the uncertainties due to self-absorption are considered. The parallel slopes of the Boltzmann plots observed for both times prove the high accuracy of the temperature measurements (of a few $\%$ ).

The electron density $n_{e}$ was deduced from Stark broadening of the spectral lines listed in Table 2 assuming a linear dependence of the Stark width on $n_{e}$ [24]. The applied Stark broadening parameters were measured in separate experiments $[25,26]$. The list of spectral lines from nickel atoms is completed by the Ar I $826.45 \mathrm{~nm}$ transition. The electron density

Table 2: Transitions used for $n_{e}$-measurements: wavelength $\lambda$, transition probability $A_{u l}$, energy $E$ and total angular momentum quantum number $J$ of upper (index $u$ ) and lower (index $l$ ) excitation levels, Stark width $w$ and shift $d$ for $n_{e}=1 \times 10^{17} \mathrm{~cm}^{-3}$.

\begin{tabular}{ccrrrrrrc}
\hline \multicolumn{1}{c}{$\begin{array}{c}A_{l} \\
(\mathrm{~nm})\end{array}$} & $\begin{array}{r}A_{u l} \\
\left(\mu s^{-1}\right)\end{array}$ & $\begin{array}{c}E_{l} \\
(\mathrm{eV})\end{array}$ & $\begin{array}{c}E_{u} \\
(\mathrm{eV})\end{array}$ & $J_{l}$ & $J_{u}$ & $\begin{array}{c}w \\
(\mathrm{pm})\end{array}$ & $\begin{array}{c}d \\
(\mathrm{pm})\end{array}$ \\
\hline Ni I & 341.476 & 55.00 & 0.03 & 3.66 & 7 & 9 & $7.6^{a}$ & $0.4^{a}$ \\
Ni I & 501.757 & 20.00 & 3.54 & 6.01 & 11 & 11 & $50^{b}$ & $18^{b}$ \\
Ni I & 503.536 & 57.00 & 3.64 & 6.10 & 7 & 9 & $200^{b}$ & $-40^{b}$ \\
Ni I & 547.690 & 9.50 & 1.83 & 4.09 & 1 & 3 & $18^{b}$ & $6^{b}$ \\
Ni I & 712.219 & 21.00 & 3.54 & 5.28 & 5 & 7 & $170^{b}$ & $80^{b}$ \\
Ar I & 826.452 & 15.30 & 11.83 & 13.33 & 3 & 3 & $98^{a}$ & $6^{b}$
\end{tabular}

$a$ Ref. [23].

${ }^{b}$ measured. 
deduced from Stark broadening of this argon transition was found to match closely (within 10\%) the values obtained with the nickel lines. This indicates that the electron density within the background gas volume close to the vapor-gas contact front equals the $n_{e}$-value within the ablated vapor plume.

The measured values of electron density and temperature are displayed in Fig. 2 as functions of time on a double logarithmic scale. They were approximated by functions $n_{e}(t)=2.19 \times 10^{20} t^{-1.067}$ and $T(t)=4.93 \times 10^{4} t^{-0.220}$, where $n_{e}, T$ and $t$ are in units of $\mathrm{cm}^{-3}, \mathrm{~K}$ and $\mathrm{ns}$, respectively. The functions are close to the evolutions $n_{e}(t)$ and $T(t)$ observed for laser ablation of steel under similar experimental conditions [10]. According to their large number of excitation levels, separated by relative small energy gaps, the metallic plasma species favor the establishment of local thermodynamic equilibrium. It is therefore reasonable to expect LTE conditions until a time of the order of $10 \mu \mathrm{s}$ as confirmed by the Boltzmann plots that cover the entire time-interval of measurements.

\subsection{Observation of blackbody radiation}

The accurate diagnostics of the plasma allowed us to retrieve the elemental composition of the ablated nickel-chromium-molybdenum alloy (see Table 1). For details of the calibration-free (CF) LIBS measurement algorithm, we refer to previous work [19, 20].

In opposition to CF-LIBS analyses based on the optically thin hypothesis (standard CF-LIBS approach proposed by Ciucci et al. [27]), calibration-free LIBS measurements that account for self-absorption require the knowledge of the plasma size along the line of sight

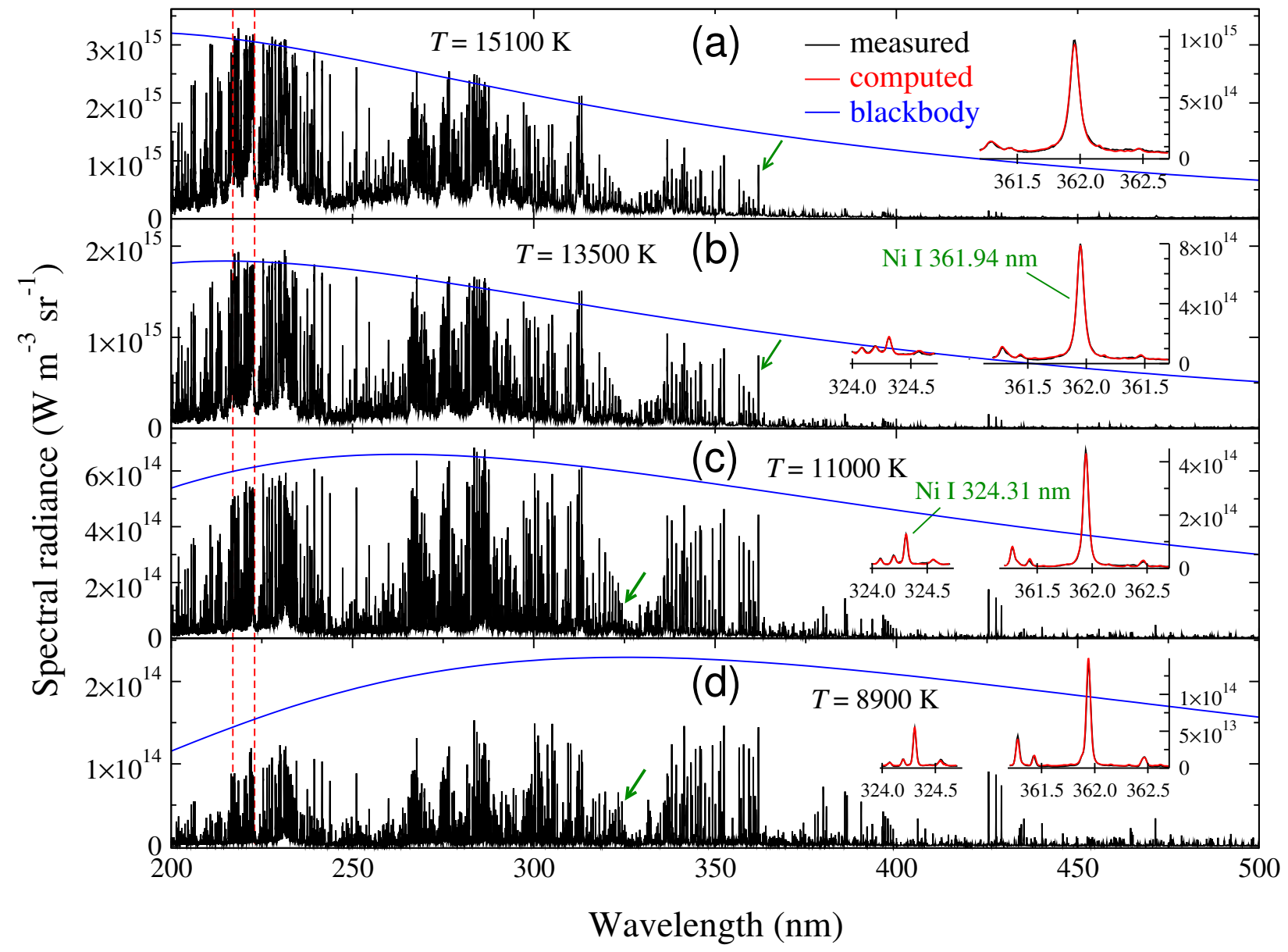

Figure 3: Spectra recorded for $t=230 \mathrm{~ns}$ (a), $350 \mathrm{~ns}$ (b), $850 \mathrm{~ns}$ (c) and $2500 \mathrm{~ns}$ (d). The transition used to adjust the measured spectrum to the computed radiance (see arrow) is displayed in the inset. The most intense lines are shown to saturate at the blackbody spectral radiance for $t<1 \mu$ s. The temperature was deduced from the intensity distribution of transitions having weak optical thicknesses. The dashed lines indicate the narrow spectral range for which measured and computed spectra are displayed in Fig. 4. 
[see Eq. (1)]. The $L$-value can be obtained either from plume imaging or from the simultaneous observation of spectral lines having significantly different optical thicknesses [19, 10].

To illustrate this, we may consider the two opposite cases of completely thin and thick spectral lines. In the optically thin case $(\tau<<1)$, Eq. (1) simplifies to $B_{\lambda}^{\text {thin }}=\varepsilon_{\lambda} L$. The line intensity increases linearly with the plasma size along the line of sight. In the opposite case, Eq. (2) applies and the spectral line intensity becomes independent of $L$. Thus, after the evaluation of temperature, electron density and relative elemental fractions (and thus of all species densities), the plasma size along the line of sight can be deduced. Once, $T$, $n_{e}$, the relative elemental fractions and $L$ are known, we can compare the computed spectral radiance to the measured spectrum.

Spectra recorded for different times are displayed in Fig. 3. For each spectrum, the transition used to adjust the intensity of the measured spectrum to the computed radiance is shown in the inset. As the optical thickness of the Ni I $361.94 \mathrm{~nm}$ transition is large at later times [(c) and (d)], the Ni I $324.31 \mathrm{~nm}$ line of significantly smaller optical thickness is also displayed. A good agreement between measured and computed line profiles is observed for both transitions, showing that self-absorption is accurately taken into account. The intensity adjustment is equivalent to an intensity calibration and the measured spectrum can thus be presented in units of spectral radiance. This allows us to compare the measured spectra to the blackbody radiation.

The most intense spectral lines are shown to saturate at the blackbody spectral radiance in agreement with the model of a uniform plasma in local thermodynamic equilibrium [Eqs. (1) and (2)]. These lines belong to $\mathrm{Ni}^{+}$ions. With increasing time, the number densities of ions decrease due to recombination and the optical thickness of $\mathrm{Ni}^{+}$transitions diminishes. The number of spectral lines that saturate at the blackbody radiance is therefore larger at early times and progressively reduces with increasing $t$. For the early delays [(a) and (b)], the lines that reach the blackbody level are distributed over a spectral window of about $100 \mathrm{~nm}$ width, allowing us to follow the change of the blackbody intensity distribution with temperature.

We emphasize that the blackbody temperature equals the electronic excitation temperature deduced from the Boltzmann plots (see Figs. 1 and 2). The equality of both temperatures is a direct proof of the establishment of local thermodynamic equilibrium in the laser-produced plasma.

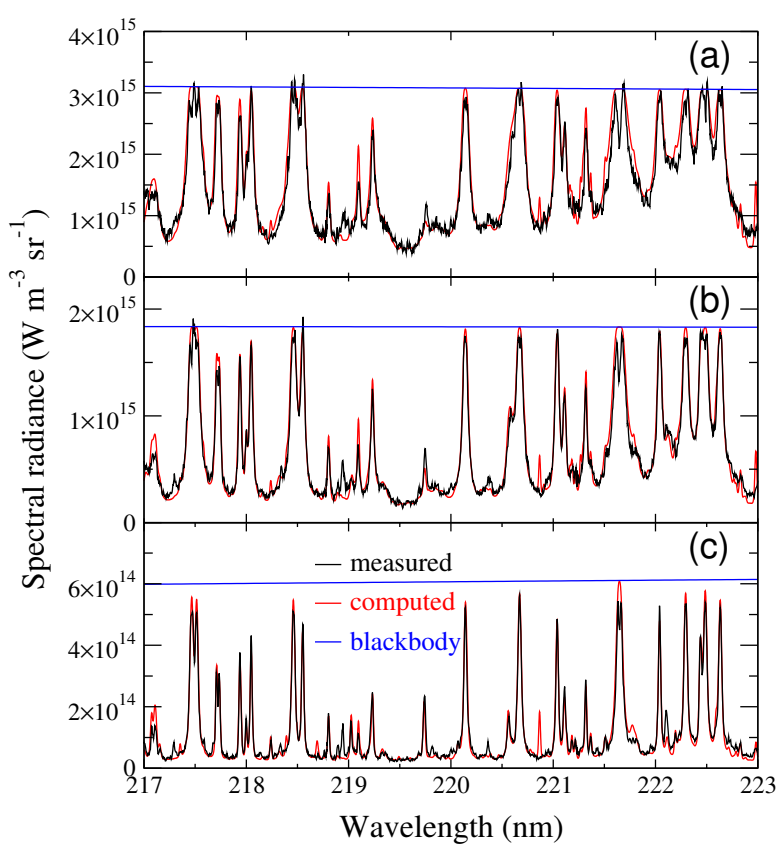

Figure 4: Narrow range of spectra displayed in Fig. 3 for $t=230 \mathrm{~ns}$ (a), $350 \mathrm{~ns}$ (b), and $850 \mathrm{~ns}$ (c). Comparison to the computed spectral radiance. The strongest $\mathrm{Ni}^{+}$-transitions are shown to saturate at the blackbody spectral radiance.

To clearly illustrate the progressive decrease of the optical thickness of $\mathrm{Ni}^{+}$transitions, the measured spectra are displayed in Fig. 4 together with the computed spectral radiance for a narrow spectral window including several of the strongest lines. The simulations show that the most intense lines saturate at the blackbody radiance only at short times, when the corresponding population number densities are large enough. Compared to simulations of emission spectra recorded during laser ablation of steel [12, 10], the agreement between measured and computed spectra is less good. This is attributed to the quality of available spectroscopic data. Compared to iron, the transition probabilities of $\mathrm{Ni}, \mathrm{Cr}$, Mo and $\mathrm{Nb}$ have much larger uncertainties [21]. However, the reduced accuracy does not affect the conclusions of the present work. The saturation at the blackbody radiance occurs independently of the transition probability if the optical thickness is large enough.

\section{Conclusion}

The time-resolved spectroscopic measurements of the plasma produced by material ablation with ultraviolet nanosecond laser pulses in an argon atmosphere al- 
lowed us to observe the saturation of the strongest emission lines at the blackbody radiance. The demonstration is given for ablation of a nickel-chromium-molybdenum alloy that generates a very rich emission spectrum with many strong transitions. To enable accurate plasma diagnostics, the large uncertainties of available spectroscopic data were compensated by the use of a large number of spectral lines for temperature and electron density measurements.

It is shown that the excitation temperature of atoms and ions equals the blackbody temperature. This is an alternative and direct proof of the establishment of local thermodynamic equilibrium. A major consequence of this demonstration is that the plasma produced under the present experimental conditions can be described by simple and accurate modeling. The clear evidence of LTE re-opens the perspectives of quantitative material analysis via calibration-free laser-induced breakdown spectroscopy.

From a practical point of view, the saturation of transitions at the blackbody spectral radiance can be exploited for the intensity calibration of spectroscopic instruments. Moreover, we anticipate that the intense continuum emission generated during the early expansion stage of the laser-induced plasma may serve as a radiation standard. This point, however, requires further investigations of the plasma properties in the initial timeinterval.

\section{Acknowledgements}

The authors acknowledge Arnaud Bultel for fruitful discussions on equilibrium radiation sources. The research leading to these results has received funding from LASERLAB-EUROPE (Grant Agreement No. 654148, European Union's Horizon 2020 research and innovation programme). EA and VC acknowledge the financial support from Romanian national projects NUCLEU-LAPLAS and PCCA 226.

[1] H. R. Griem, Plasma spectroscopy, Academic, New York, 1964

[2] R. McWhirter, Ch. 5, in: R. H. Huddlestone (Ed.), Plasma Diagnostic Techniques, Academic, New York, 1965, pp. $201-264$.

[3] J. Hermann, C. Dutouquet, Local thermal equilibrium plasma modeling for analyses of gas-phase reactions during reactivelaser ablation, J. Appl. Phys. 91 (2002) 10188-10193.

[4] W. L. Wiese, Spectroscopic diagnostics of low temperature plasmas: techniques and required data, Spectrochim. Acta Part B: Atom. Spectrosc. 46B (6/7) (1991) 831-841.

[5] T. Belmonte, C. Noël, T. Gries, J. Martin, G. Henrion, Theoretical background of optical emission spectroscopy for analysis of atmospheric pressure plasmas, Plasma Sources Sci. Technol. 24 (2015) 064003 1-29.
[6] D. W. Hahn, N. Omenetto, Laser-induced breakdown spectroscopy (LIBS), Part I: Review of basic diagnostics and plasmaparticle interactions: still-challenging issues within the analytical plasma community, Appl. Spectrosc. 64 (2010) 335A-366A.

[7] G. Cristoforetti, E. Tognoni, L. A. Gizzi, Thermodynamic equilibrium states in laser-induced plasmas: From the general case to laser-induced breakdown spectroscopy plasmas, Spectrochim. Acta Part B: Atom. Spectrosc. 90 (2013) 1-22.

[8] A. D. Giacomo, J. Hermann, Laser-induced plasma emission: from atomic to molecular spectra, J. Phys. D: Appl. Phys. 50 (2017) $1830021-17$.

[9] S. V. Shabanov, I. B. Gornushkin, Two-dimensional axisymmetric models of laser induced plasmas relevant to laser induced breakdown spectroscopy, Spectrochim. Acta Part B: Atom. Spectrosc. 100 (2014) 147-172.

[10] J. Hermann, E. Axente, V. Craciun, A. Taleb, F. Pelascini, Evaluation of pressure in a plasma produced by laser ablation of steel, Spectrochim. Acta Part B: Atom. Spectrosc. (2018) accepted for publication on February 24th

[11] J. Hermann, C. Gerhard, E. Axente, C. Dutouquet, Comparative investigation of laser ablation plumes in air and argon by analysis of spectral line shapes: Insights on calibration-free laserinduced breakdown spectroscopy, Spectrochim. Acta Part B: Atom. Spectrosc. (2014) 189-196.

[12] J. Hermann, D. Grojo, E. Axente, C. Gerhard, M. Burger, V. Craciun, Ideal radiation source for plasma spectroscopy generated by laser ablation, Phys. Rev. E 96 (2017) 053210 1-6.

[13] J. Cooper, Plasma spectroscopy, Rep. Prog. Phys. 29 (1966) 35130

[14] C. Gerthsen, H. O. Kneser, H. Vogel, Physik, Springer, Berlin, 1986.

[15] C. R. Darling, Pyrometry, Spon LTD, London, 1920.

[16] J. C. Morris, R. L. Garrison, A radiation standard for the vacuum violet, J. Quant. Spectrosc. Radiat. Transf. 9 (1969) 1407-1418.

[17] R. Lincke, H. R. Griem, Method for the determination of atomic-resonance line-oscillator strengths from widths of optically thick emission lines in t-tube plasmas, Phys. Rev. 143 (1966) 66-73.

[18] J. Hermann, C. Boulmer-Leborgne, D. Hong, Diagnostics of the early phase of an ultraviolet laser induced plasma by spectral line analysis considering self-absorption, J. Appl. Phys. 83 (2) (1998) 691-696.

[19] C. Gerhard, J. Hermann, L. Mercadier, L. Loewenthal, E. Axente, C. R. Luculescu, T. Sarnet, M. Sentis, W. Viöl, Quantitative analyses of glass via laser-induced breakdown spectroscopy in argon, Spectrochim. Acta Part B: Atom. Spectrosc. 101 (2014) 32-45.

[20] J. Hermann, A. Lorusso, A. Perrone, F. Strafella, C. Dutouquet, B. Torralba, Simulation of emission spectra from nonuniform reactive laser-induced plasmas, Phys. Rev. E 92 (2015) 053103 $1-15$.

[21] A. Kramida, Y. Ralchenko, J. Reader, NIST Atomic Spectra Database (version 5.3), National Institute of Standards and Technology, Gaithersburg, MD (2015). URL http://physics.nist.gov/asd

[22] P. L. Smith, C. Heise, J. R. Esmond, R. L. Kurucz, Atomic spectral line database built from atomic data files from R. L. Kurucz CD-ROM 23 (2011). URL http://www.pmp. uni-hannover.de/cgi-bin/ssi/test/kurucz/seku

[23] N. Konjević, A. Lesage, J. R. Führ, W. L. Wiese, Experimental Stark widths and shifts for spectral lines of neutral and ionized atoms (A critical review of selected data for the period 1989 through 2000), J. Phys. Chem. Ref. Data 31 (2002) 819-921.

[24] E. Tognoni, V. Palleschi, M. Corsi, G. Cristoforetti, N. Omenetto, I. Gornushkin, B. W. Smith, J. D. Wineford- 
ner, From sample to signal in laser-induced breakdown spectroscopy: a complex route to quantitative analysis, in: A. W. Miziolek, V. Palleschi, I. Schechter (Eds.), Laser-induced breakdown spectroscopy, Cambridge University, Berlin, 2006, pp 122-194.

[25] M. Cirisan, M. Cvejić, M. R. Gavrilović, S. Jovicević, N. Konjević, J. Hermann, Stark broadening measurement of $\mathrm{Al}$ II lines in a laser-induced plasma, J. Quant. Spectrosc. Radiat. Transfer 133 (2014) 652-662.

[26] M. Burger, J. Hermann, Stark broadening measurements in plasmas produced by laser ablation of hydrogen containing compounds, Spectrochim. Acta Part B: Atom. Spectrosc. 122 (2016) 118-126.

[27] A. Ciucci, M. Corsi, V. Palleschi, S. Rastelli, A. Salvetti, E. Tognoni, New procedure for quantitative elemental analysis by laser-induced plasma spectroscopy, Appl. Spectrosc. 53 (1999) 960-964. 